\title{
A checklist of traditional edible bio-resources from Ima markets of Imphal Valley, Manipur, India
}

\author{
Oinam Sunanda Devi ${ }^{1}$, Puspa Komor ${ }^{2} \&$ Dhritiman Das ${ }^{3}$ \\ ${ }^{1}$ Research Scholar, Department of Zoology, Gauhati University, Guwahati, Assam 781014, India \\ ${ }^{2}$ Junior Research Fellow, Omeo Kumar Das Institute of Social Change and Development (OKDISCD), VIP Road, Upper Hengrabari, \\ Guwahati, Assam 781036, India \\ ${ }^{3} \mathrm{PhD}$ Scholar, Conservation Science, Ashoka Trust for Research in Ecology and the Environment (ATREE), 5th 'A' Main Road, \\ Hebbal, Bengaluru, Karnataka 560024, India \\ Email: ${ }^{1}$ sunan_o@rediffmail.com, ${ }^{2}$ puspakomorch@gmail.com, ${ }^{3}$ dhritiman.das@atree.org
}

Abstract: A study was conducted at seven important markets of Imphal Valley, Manipur in northeastern India, which are run exclusively by women and are popularly known as "the Ima markets". The two year study was to find out the important edible bio-resources which are consumed daily by the loca people of Manipur. Regular surveys were conducted at the selected markets at least three times a month. A total of 45 wild edible plants and 26 wild fruits were identified during the survey. Also, 25 edible animal resources were recorded. It is suggested that the high diversity of wild edible bio-resources needs to be conserved for livelihood sustenance of the local communities.

Keywords: Bio-resource, edible, Ima Market, Imphal Valley, livelihood, Manipur.

Manipur forms a distinctive part of Indo-Burma inhabited by various ethnic groups and communities possessing varied cultures and traditions (Singh et al. 1996). It lies in the extreme east of India bordering Myanmar within $23^{\circ} 47-25^{\circ} 41^{\prime} \mathrm{N}$ \& $93^{\circ} 61^{\prime} \mathrm{E}-94^{\circ} 48^{\prime} \mathrm{E}$ (Singh et al. 1996). The terrain of the state is predominantly hilly $(92 \%)$, except for a broad alluvial valley, called the

Date of publication (online): 26 October 2010

Date of publication (print): 26 October 2010

ISSN 0974-7907 (online) | 0974-7893 (print)

Editor: Anwaruddin Choudhury

Manuscript details:

Ms \# 02256

Received 09 July 2009

Final revised received 31 August 2010

Finally accepted 06 September 2010

Citation: Devi, O.S., P. Komor \& D. Das (2010). A checklist of traditiona edible bio-resources from Ima markets of Imphal Valley, Manipur, India. Journal of Threatened Taxa 2(11): 1291-1296.

Copyright: (c) Oinam Sunanda Devi, Puspa Komor \& Dhritiman Das 2010. Creative Commons Attribution 3.0 Unported License. JoTT allows unrestricted use of this article in any medium for non-profit purposes, reproduction and distribution by providing adequate credit to the authors and the source of publication.

Acknowledgements: We like to thank Prof. P.C. Bhattacharjee and Dr. P.K. Saikia, Department of Zoology, Gauhati University for their help and guidance during the survey period. We also like to thank our family members for their financial help and support during the survey work.

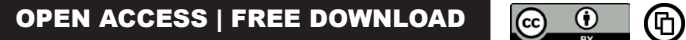

Imphal Valley, which extends north-south in the middle for over $1795 \mathrm{~km}^{2}$ (Singh et al. 1996). The Imphal Valley forms only $8.25 \%$ of the total area of Manipur while the remaining $91.75 \%$ is hills (Singh 2006). Although the valley constitutes only a small part of the geographical area, two-thirds of Manipur's 1.8 million people live in the Imphal Valley (Roy 1992).

The state falls in the Indo-Burma global biodiversity hotspot (Myers et al. 2000; Mittermeier et al. 2004). Its rich bio-resources have acted as the life source of different indigenous communities living in harmony within the area for centuries. The dominant indigenous communities of Manipur include the Meitei, Naga, Kuki and other small communities like Manipuri Muslim and Nepali people (Elangbam 2002). Most of these people depend directly or indirectly on wild bio-resources for their livelihood. The local people collect different edible wild bio-resources from the nearby hills and plains for their own food and also to sell them in the markets. One important feature of Manipur is that women play a very important role in the society both socially and economically. It is worth mentioning that most of the markets in the Imphal valley are run and controlled by women, these are popularly known as the Ima Market or Mother's Market. Ima means mother in the local language of Manipur. The main Ima Market is located at Khwairamban Bazaar in the heart of the city where more than 3000 women from different communities congregate everyday to sell local vegetables, fish, grain, handlooms, handicrafts and other household items. The uniqueness of Ima market is that it is exclusively for women and one will not find a single man doing business in the market or its vicinity. Ima (Mother), Ine (Aunt) and Iche (Sister) from various communities run the marketplace to support their families.

Through this study an attempt has been made to list the important edible bio-resources sold and consumed by the local people of the state so as to identify the rare and endemic bio-resources for their future conservation.

Study area: The present study was conducted at seven important Ima markets of Imphal valley run. The market places are Khwairamban Bazaar, Singjamei Bazaar, Kwakeithel Bazaar, Tera Bazaar situated at 
Imphal West District; Khurai Lamlong Bazaar, Kongba Bazaar and Chingmeirong Bazaar situated at Imphal East District. The market places are locally called "Bazaars" in Manipur. The Khwairamban Bazaar is the biggest market of Imphal situated in the heart of the city where more than 3000 women vendors sell vegetables, fruits, fishes and daily household items under rows of small markets built together. The Singjamei Bazaar is the second largest bazaar run by nearly 1000 women vendors. The other five bazaars are smaller in size, run by about 500 women each. There is no fixed size of the markets and the number of women vendors also fluctuates daily. Sometimes new women vendors do not get a permanent place inside the market place so they sell their items on the roadsides thus expanding the markets. At other times permanent vendors come out to the roadside as some people buy things in a hurry and do not want to bother roaming inside the markets. Women of different communities of the state run the markets everyday from early morning around $0500 \mathrm{hr}$ up to $1900 \mathrm{hr}$ under normal circumstances. The markets are run very systematically, all the items are sold separately and there is no mix of things reducing the confusion of the buyers and also reducing congestion. The frequent bandhs and general strikes called by different organizations in the state greatly affect the economy as well as the livelihood of these thousands of women from far flung areas of the state.

Methods: The methods employed in this study were so designed to collect information on the wild plants and animal bio-resources which are consumed daily by the local people of Manipur. Literatures were thoroughly searched for references on the subject and field data was collected for a period of two years from January 2007 to December 2008. Markets were surveyed and extensive interviews of women vendors were taken with regard to availability of wild bio-resources, following Jain (1989, 1990) and Martin (1995).

Regular surveys were conducted at the seven selected markets at least two times a month. Surveys were conducted at the peak marketing hours between 0700-1000 hr and 0300-1900 hr. At least 1-2 hours was spent in each market during the data collection. A total of 48 days and 144 hours were spent in each market place during the two years field surveys. A minimum of 100 women vendors in each market were interviewed regarding the local name of the bio-resource, their use, source, price and place from where they brought the items. All the edible wild plants, fruits and animal products were collected and identified with the help of specialists and relevant published papers and books such as Singh \& Arora (1978), Sukla et al. (1982), Jhingran et al. (1991), Jain (1999), Elangbam (2002), Singh (2002), Khanna (2003), and Vishwanath \& Geetakumari (2009). The bioresources were categorized crudely as common or rare as per their availability and encounter rates in the market places.
Results: A total of 45 wild edible plants including two mushroom and two edible fungus varieties were identified during the surveys. The study also records 26 wild fruits and 25 edible animal bio-resources including two eel varieties, three gastropod varieties, one bivalve variety, one crustacean variety and 18 local fresh water fish varieties.

Out of the 45 edible plants, 37 are common species and eight are rare species. All the plants are seasonal i. e. $35.5 \%$ are summer species, $55.5 \%$ are winter species and $8.9 \%$ are found throughout the year. Some Rare plants are scarcely available and as a result their price is also high. For example Pleurotus ostreatus, a mushroom variety locally called "Uyen" costs Rs. $60-80 / \mathrm{kg}$ because of its high demand and high nutritive value. Some plant species are abundantly available so they are cheaper. For example Oenunthe javanica, a local herb variety found in open fields and wetlands called locally "Komprek", costs only Rs. $10-20 / \mathrm{kg}$.

The study also records 26 local wild fruit varieties, of which 19 are Common and seven are rare varieties. $53.8 \%$ of these are summer varieties, $38.5 \%$ are winter varieties and $7.7 \%$ are year round varieties.

Of the total 25 edible animal bio-resources, 17 are common species and eight are rare species. These includes two freshwater eel species Monopterus albus and Anguilla bengalensis, three gastropod species Pila globossa, Pila spp. and Turittella spp., one fresh water mussel species Anodonta anatina, one freshwater crustacean species Macrobrachium dayanum and 18 fresh water fish species. Out of the total species $16 \%$ are found in summer, 52\% are found in winter and $32 \%$ are found throughout the year.

Of the 100 women vendors interviewed in each market, $42.4 \%$ of the respondents said that they had collected the bio-resources themselves and $57.6 \%$ revealed that they had collected the resources from one or two local distributors.

The lists of all bio-resources are given below in the Tables 1, 2 and 3.

Discussion: From time immemorial, the indigenous local inhabitants of Manipur have had a wide knowledge about the uses of traditional bio-resources, either as food or medicine in their day-to-day life (Khumbongmayum et. al. 2004). In this study, a total of 45 wild edible plants, 26 wild fruit varieties and 25 edible animal bio-resources were recorded, all of which form an important part in the diet of the local Manipuri people. The local peoples mainly the women of different castes and communities went to far-flung areas and into the forests to collect these edible bio-resources for their own food and to sell them in the local markets as a source of income for themselves. Some wild edible plants are rich in nutrient content, and may even be superior to cultivated ones in this respect (Sundriyal \& Sundriyal 2001a). A varied diet of wild edible plants may also increase one's sense of food palatability, 
Table 1. Edible wild local plants found in the markets of Imphal Valley

\begin{tabular}{|c|c|c|c|c|c|c|c|}
\hline & Family & Scientific name & Local name & Season & Status & $\begin{array}{l}\text { Average } \\
\text { price (Rs) }\end{array}$ & Source \\
\hline 1 & Polygonaceae & Polygonium barbatum Linn. & Yelang & W & $\mathrm{C}$ & $20-25 / \mathrm{Kg}$ & Open fields \\
\hline 2 & Plumbaginaceae & Phumbago zeylanica Linn. & Kengoi & W & $\mathrm{C}$ & $15-20 / \mathrm{Kg}$ & Open fields \\
\hline 3 & Zingiberaceae & Cucurma angustifolia Roxb. & Yaipal & W & $\mathrm{C}$ & $30-40 / \mathrm{Kg}$ & Hill forests \\
\hline 4 & Zingiberaceae & Hedychium coronarium Koening. & Loklei & $S$ & $\mathrm{C}$ & $20-25 / \mathrm{Kg}$ & Forests, open fields \\
\hline 5 & Zingiberaceae & Alpinia nigra (Gaertn) Burtt. & Pullei & $S$ & $\mathrm{C}$ & $40-50 / \mathrm{Kg}$ & Forests, open fields \\
\hline 6 & Poaceae & Zizania latifolia (Turez.Ex. Stapf) & Eshing Kambong & $\mathrm{S}$ & $\mathrm{C}$ & $20-25 / \mathrm{Kg}$ & Wetlands \\
\hline 7 & Apiaceae & Oenunthe javanica (Blume) DC. & Komprek & W & $\mathrm{C}$ & $10-15 / \mathrm{Kg}$ & $\begin{array}{l}\text { Marshes, open fields, } \\
\text { wetlands }\end{array}$ \\
\hline 8 & Comolvulaceae & Ipomea aquatica Forsk. & Kollamni & W & $\mathrm{C}$ & $15-20 / \mathrm{Kg}$ & Wetlands \\
\hline 9 & Araceae & Alocasia cucullata (L) Schott. & Singjupal & YR & $\mathrm{C}$ & $10-20 / \mathrm{Kg}$ & $\begin{array}{l}\text { Marshes, wetlands, } \\
\text { open fields }\end{array}$ \\
\hline 10 & Cyperaceae & Cyperus esculentus, L. & Kaothum & W & C & $10-20 / \mathrm{Kg}$ & $\begin{array}{l}\text { Paddyfields and open } \\
\text { fields }\end{array}$ \\
\hline 11 & Euphorbiaceae & Antiderma diaindrum (Roxb.) Roth. & Ching Yensin & W & $\mathrm{C}$ & $10-15 / \mathrm{kg}$ & Hills \\
\hline 12 & Apiaceae & Ceutella asiatica (L.) & Peruk & W & $\mathrm{C}$ & $20-30 / \mathrm{Kg}$ & Open fields \\
\hline 13 & Trapaceae & Trapa natans $\mathrm{L}$. & Heikak yeli & W & $\mathrm{C}$ & $10-15 / \mathrm{Kg}$ & Lakes \& wetlands \\
\hline 14 & Nymphaeaceae & Eurgale ferox, Salisb. & Thanging & $S$ & $\mathrm{C}$ & $30-50 / \mathrm{Kg}$ & Wetlands, ponds etc \\
\hline 15 & Nymphaeaceae & Nelumbo nucifera (Gaertn) & Thambou & W & C & $20-30 / \mathrm{Kg}$ & $\begin{array}{l}\text { Wetlands, ponds, } \\
\text { lakes }\end{array}$ \\
\hline 16 & Nymphaeaceae & Nymphaea nouchali Burm.f. & Tharo Mari & W & $\mathrm{C}$ & $25-30 / \mathrm{Kg}$ & $\begin{array}{l}\text { Wetlands, ponds, } \\
\text { lakes }\end{array}$ \\
\hline 17 & Alismataceae & Sagittaria sagiltifolia Linn. & Koukha & W & $\mathrm{C}$ & $40-50 / \mathrm{Kg}$ & Open fields \\
\hline 18 & Cycadaceae & Cycas pectinata Grift. & Yendang & W & $\mathrm{C}$ & $20-25 / \mathrm{kg}$ & Marshes, hills \\
\hline 19 & Mimosaceae & Neptunia oleraceae Lous. & Ekai thibi & W & $\mathrm{C}$ & $20-30 / \mathrm{kg}$ & Ponds, wetlands \\
\hline 20 & Chenopodiaceae & Chenopodium album Linn. & Monsaobi & W & $\mathrm{C}$ & $15-20 / \mathrm{Kg}$ & Marshes, openfields \\
\hline 21 & Lamiaceae & Meriandra bengalensis Benth. & Kanghuman & $\mathrm{S}$ & $\mathrm{C}$ & $10-15 / \mathrm{Kg}$ & Openfields \\
\hline 22 & Palilionaceae & Sesbania sesban (Jacq.) W. Wight & Chuchurangmei & $S$ & $\mathrm{C}$ & $20-30 / \mathrm{Kg}$ & Open fields, hills \\
\hline 23 & Vitaceae & Cessins javana Linn. & Kongouyen & $\mathrm{S}$ & $\mathrm{C}$ & $20-25 / \mathrm{Kg}$ & Open fields \\
\hline 24 & Moraceae & Ficus palmate Linn & Heiban & W & $\mathrm{C}$ & $20-25 / \mathrm{kg}$ & Hills \\
\hline 25 & Rubiaceae & Vanguirea speniosa Linn & Heibi mana & W & $\mathrm{C}$ & $15-20 / \mathrm{Kg}$ & Hills, open fields \\
\hline 26 & Caryophyllaceae & Stelaria media Linn. & Yerum keirum & W & $\mathrm{C}$ & $20-25 / \mathrm{Kg}$ & Open fields \\
\hline 27 & Amaranthaceae & Amaranthus viridis $\mathrm{L}$. & Chengkruk & W & $\mathrm{R}$ & $25-30 / \mathrm{Kg}$ & Hills \\
\hline 28 & Graminaceae & Ustilago consimilis Sydow & Kambong & W & $\mathrm{C}$ & $25-40 / \mathrm{Kg}$ & Wetlands \\
\hline 29 & Polyporaceae & $\begin{array}{l}\text { Pleurotus ostreatus (Jacquin ex. } \\
\text { Fr) Kummer. }\end{array}$ & Uyen & $S$ & $\mathrm{R}$ & $60-80 / \mathrm{Kg}$ & Hills \\
\hline 30 & Schizophyllaceae & Schizophyllum commune, Fr. & Kanglayen & $S$ & $\mathrm{R}$ & $25-50 / \mathrm{Kg}$ & Hills \\
\hline 31 & Auriculariaceae & Auricularia delicata (Fr.) P.Henn. & Uchina & $S$ & $\mathrm{C}$ & $20-30 / \mathrm{Kg}$ & Hills \\
\hline 32 & Agaricaceae & Agaricus campestris L.ex. Fr. & Chengum & W & $\mathrm{C}$ & $50-60 / \mathrm{Kg}$ & $\begin{array}{l}\text { Hills, river } \\
\text { embankments. }\end{array}$ \\
\hline 33 & Alismataceae & Alisma plantago-aquatica Linn. & Ishing Yempat & $S$ & $\mathrm{C}$ & $10-15 / \mathrm{Kg}$ & Wetlands \\
\hline 34 & Araceae & Colocasia antiquorium (L) Scott & Lam Pal & YR & $\mathrm{C}$ & $! 5-20 / \mathrm{Kg}$ & $\begin{array}{l}\text { Marshes and open } \\
\text { fields }\end{array}$ \\
\hline 35 & Malvaceae & Hibiscus cannabinus Linn. & Shougree & YR & $\mathrm{C}$ & $10-20 / \mathrm{Kg}$ & Open fields \\
\hline 36 & Malvaceae & Hibiscus sabdariffa Linn. & Shilhot Shougree & YR & $\mathrm{C}$ & $10-25 / \mathrm{Kg}$ & Open fields \\
\hline 37 & Saururaceae & Houttuynia cordata Thunb. & Tokning- khok & W & $\mathrm{C}$ & $10-15 / \mathrm{kg}$ & Open fields \\
\hline 38 & Lamiaceae & Meriandra benghalensis Benth. & Kanghoo- man & W & $\mathrm{C}$ & $10-20 / \mathrm{Kg}$ & Open fields \\
\hline 39 & Compositae & Enhydra flactuans Lour. & Komprek Tujombi & W & $\mathrm{R}$ & $20-30 / \mathrm{kg}$ & Wetlands \\
\hline
\end{tabular}




\begin{tabular}{|c|c|c|c|c|c|c|c|}
\hline & Family & Scientific name & Local name & Season & Status & $\begin{array}{c}\text { Average } \\
\text { price (Rs) }\end{array}$ & Source \\
\hline 40 & Chenopodiaceae & Chenopodium album Linn & Monsaobi & S & $\mathrm{C}$ & $20-30 / \mathrm{kg}$ & Open fields \\
\hline 41 & Saururaceae & Houttuynia cordata Thunb. & Tokningkok & S & $\mathrm{C}$ & $10-20 / \mathrm{kg}$ & Open fields \\
\hline 42 & Oxalidaceae & Oxalis corniculata Linn. & Yensil & S & $\mathrm{R}$ & $20-30 / \mathrm{kg}$ & Open fields \& hills. \\
\hline 43 & Polygonaceae & Rumex maritimus Linn. & $\begin{array}{l}\text { Torong } \\
\text { Khongchak }\end{array}$ & W & $\mathrm{R}$ & $30-40 / \mathrm{kg}$ & Hills \\
\hline 44 & Polygonaceae & Polygonum chinense Linn & Angom Yensil & $\mathrm{s}$ & $\mathrm{R}$ & $20-30 / \mathrm{kg}$ & Hills \\
\hline 45 & Polygonaceae & Polygonum molle D. Don. & Tharam & $\mathrm{s}$ & $\mathrm{R}$ & $20-30 / \mathrm{kg}$ & hills \\
\hline
\end{tabular}

S - Summer; W - Winter; YR - Year round; C - Common; R - Rare.

Table 2. Edible wild local fruits found in the markets of Imphal Valley

\begin{tabular}{|c|c|c|c|c|c|c|c|}
\hline & Family & Scientific name & Local name & Season & Status & $\begin{array}{c}\text { Average } \\
\text { price (Rs) }\end{array}$ & Source \\
\hline 1 & Anacardiaceae & Rhus hookerii Sahni \& Bahadur. & Heimang & W & $\mathrm{R}$ & $40-50 / \mathrm{kg}$ & Hills, plains \\
\hline 2 & Nymphaeaceae & Nymphaea rubra Roxb. & Lemphu & W & $\mathrm{C}$ & $10-20 / \mathrm{kg}$ & Wetlands \\
\hline 3 & Clusiaceae & Garcinia pedunculata Roxb. & Heibum & W & $\mathrm{R}$ & $80-100 / \mathrm{kg}$ & Hills, plains \\
\hline 4 & Rosaceae & Prunus armeniaca L. & Malhei & W & C & $30-40 / \mathrm{kg}$ & Hills, plains \\
\hline 5 & Moraceae & Ficus cunia, Buch. Ham. Ex. Roxb. & Heirit & $S$ & $\mathrm{C}$ & $50-70 / \mathrm{kg}$ & Hills \\
\hline 6 & Myrtaceae & Elaeagnus umbellate Thunb. & Heiyai & $S$ & $\mathrm{C}$ & $30-50 / \mathrm{kg}$ & Hills, plains \\
\hline 7 & Moraceae & Morus nigra Linn. & Heijampet & $S$ & $\mathrm{R}$ & $40-50 / \mathrm{kg}$ & Hills, plains \\
\hline 8 & Myrtaceae & Syzgium cumini (L.) Skeels & Jam & $S$ & C & $10-20 / \mathrm{kg}$ & Hills, plains \\
\hline 9 & Tiliaceae & Microcos paniculata Linn. & Heitup & W & C & $30-50 / \mathrm{kg}$ & Hills \\
\hline 10 & Rubiaceae & Vanguirea spinosa Hook.f. & Heibi & W & C & $20-30 / \mathrm{kg}$ & Hills, plains \\
\hline 11 & Moraceae & Ficus glomerata Roxb. & Heibung & YR & $\mathrm{C}$ & $20-25 / \mathrm{kg}$ & Hills, plains \\
\hline 12 & Elaeocarpaceae & Elaeocarpus floribundus & Chorphon & $S$ & $\mathrm{C}$ & $20-30 / \mathrm{kg}$ & Hills, plains \\
\hline 13 & Rhamnaceae & Ziziphus mauritiana Lam. & Boroi & W & C & $20-30 / \mathrm{kg}$ & Hills, plains \\
\hline 14 & Moraceae & Artocarpus heterophyllus Lamk. & Theibong & W & C & $15-30 / p c$ & Hills, plains \\
\hline 15 & Euphorbiaceae & Phyllanthus emblica (L.) & Heikru & $\mathrm{S}$ & C & $10-30 / \mathrm{kg}$ & Hills, plains \\
\hline 16 & Averrhoaceae & Averrhoa carambola Linn. & Heinijom & W & C & $10-20 / \mathrm{kg}$ & Hills, plains \\
\hline 17 & Caesalpiniaceae & Tamarindus indica L. & Mange & YR & $\mathrm{C}$ & $10-20 / \mathrm{kg}$ & Hills, plains \\
\hline 18 & Anacardiacea & Mangifera indica Linn. & Heinou & S & $\mathrm{C}$ & $40-60 / \mathrm{kg}$ & $\begin{array}{l}\text { Open fields and } \\
\text { hills }\end{array}$ \\
\hline 19 & Rosaceae & Prunus crasoides D. Don. & Chumbrei & $\mathrm{S}$ & C & $20-30 / \mathrm{kg}$ & $\begin{array}{l}\text { Open fields \& } \\
\text { hills }\end{array}$ \\
\hline 20 & Anacardiaceae & Spondias mangifera Willd. & Heining & S & C & $20-25 / \mathrm{kg}$ & $\begin{array}{l}\text { Open field \& } \\
\text { hills }\end{array}$ \\
\hline 21 & Myristicaceae & Horsfieldia amygdalnia (Wall) Warb. & Nonganghei & $\mathrm{S}$ & $\mathrm{R}$ & $20-30 / \mathrm{kg}$ & Hills \\
\hline 22 & Dilleniaceae & Dillenia indica Linn & Heigree & $S$ & $\mathrm{C}$ & $10-20 / \mathrm{kg}$ & Foothills \\
\hline 23 & Euphorbiaceae & Baccaurea sapida (Roxb) Muell. & Motok- hei & S & $\mathrm{C}$ & $20-30 / \mathrm{kg}$ & Hills \\
\hline 24 & Meliaceae & $\begin{array}{l}\text { Aphanomixis polystachya (Wall) } \\
\text { Parker. }\end{array}$ & Heirangkhoi & $\mathrm{S}$ & $\mathrm{R}$ & $30-45 / \mathrm{kg}$ & Hills \\
\hline 25 & Moraceae & Artocarpus chaplasa Roxb. & Heirukothong & S & $\mathrm{R}$ & $20-40 / \mathrm{kg}$ & Hills \\
\hline 26 & Flacourtiacea & Flacourtia jangomas (Lour.) Raeusch. & Heitroi & $S$ & $\mathrm{R}$ & $30-40 / \mathrm{kg}$ & Hills \\
\hline
\end{tabular}

S - Summer; W - Winter; YR - Year round; C - Common; R - Rare. 
Table 3. Edible animal resource found in the markets of Imphal Valley

\begin{tabular}{|c|c|c|c|c|c|c|c|}
\hline & Family & Scientific name & Local name & Season & Status & $\begin{array}{l}\text { Average } \\
\text { price (Rs) }\end{array}$ & Source \\
\hline \multicolumn{8}{|c|}{ I. Eel Varieties } \\
\hline 1 & Synbranchidae & Monopterus albus (Zuiew) & Ngaprum & W & $\mathrm{C}$ & $150-180 / \mathrm{kg}$ & $\begin{array}{l}\text { Pond, river, } \\
\text { wetlands }\end{array}$ \\
\hline 2 & Anguillidae & Anguilla bengalensis ( Gray) & Ngaril & W & $\mathrm{R}$ & $150-250 / \mathrm{Kg}$ & River \\
\hline \multicolumn{8}{|c|}{ II. Fresh Water Fish Varieties } \\
\hline 1 & Cyprinidae & $\begin{array}{l}\text { Osteobrama belangeri } \\
\text { (Valenciennes) }\end{array}$ & Pengba & W & $\mathrm{R}$ & $300-500 / \mathrm{kg}$ & River, wetland \\
\hline 2 & Cyprinidae & Puntius sophore (Hamilton) & Phabounga & YR & $\mathrm{C}$ & $40-80 / \mathrm{kg}$ & $\begin{array}{l}\text { Wetland, lake, } \\
\text { river }\end{array}$ \\
\hline 3 & Cobitidae & $\begin{array}{l}\text { Lepidocephalus guntea (Hamilton- } \\
\text { Buchanan) }\end{array}$ & Ngakijou & W & $\mathrm{C}$ & $50-70 / \mathrm{kg}$ & River \\
\hline 4 & Bagridae & Mystus bleekeri (Day) & Ngarep & W & $\mathrm{R}$ & $40-60 / \mathrm{kg}$ & River \\
\hline 5 & Siluridae & Ompok bimaculatus (Bloch) & Ngaten & W & $\mathrm{R}$ & $80-100 / \mathrm{kg}$ & River \\
\hline 6 & Schilbeidae & Eutropiichthys vacha (Hamilton) & Ngahei & $S$ & $\mathrm{R}$ & $250-350 / \mathrm{kg}$ & Wetland, river \\
\hline 7 & Clariidae & Clarias batrachus (Linnaeus) & Ngakra & W & $\mathrm{C}$ & $100-150 / \mathrm{kg}$ & Wetland, lakes \\
\hline 8 & Heteropneustidae & Heteropneustes fossilis (Bloch) & Ngachik & YR & $\mathrm{C}$ & $60-100 / \mathrm{kg}$ & Wetland, lakes \\
\hline 9 & Belonidae & Xenentodon cancila (Hamilton) & Nga Cheklaobi & $S$ & $\mathrm{R}$ & $50-80 / \mathrm{kg}$ & Wetland \\
\hline 10 & Ambassidae & Chanda nama (Hamilton) & Nagmhai & YR & C & $30-40 / \mathrm{kg}$ & River, wetland \\
\hline 11 & Channidae & $\begin{array}{l}\text { Channa orientalis (Bloch \& } \\
\text { Schneider) }\end{array}$ & Meitei Ngamu & W & $\mathrm{C}$ & $90-120 / \mathrm{kg}$ & River, wetland \\
\hline 12 & Osphronemidae & Colisa labiosus (Day) & Pheteen & $S$ & C & $50-100 / \mathrm{kg}$ & $\begin{array}{l}\text { Wetland, river, } \\
\text { streams }\end{array}$ \\
\hline 13 & Cyprinidae & Colisa fasciatus (Bloch \& Schneider) & Ngapema & YR & $\mathrm{C}$ & $40-80 / \mathrm{kg}$ & Wetland, river \\
\hline 14 & Anabantidae & Anabas testudineus (Bloch) & Ukabi & YR & $\mathrm{C}$ & $80-100 / \mathrm{kg}$ & Wetland, river \\
\hline 15 & Bagridae & Mystus spp. & Ngachep & $S$ & $\mathrm{C}$ & $40-50 / \mathrm{kg}$ & River \\
\hline 16 & Channidae & Channa punctatus (Bloch) & Ngamu & YR & $\mathrm{C}$ & $50-80 / \mathrm{kg}$ & $\begin{array}{l}\text { River, ponds, } \\
\text { wetlands }\end{array}$ \\
\hline 17 & Channidae & Channa striatus (Bloch) & Porom & YR & $\mathrm{C}$ & $150-200 / \mathrm{kg}$ & Wetlands \\
\hline 18 & Siluridae & Wallago attu (Bloch \& Schneider) & Sareng & W & $\mathrm{R}$ & $200-300 / \mathrm{kg}$ & River \\
\hline \multicolumn{8}{|c|}{ III. Fresh Water Prawn Variety } \\
\hline 1 & Palaemonidae & $\begin{array}{l}\text { Macrobrachium dayanum. } \\
\text { (Henderson) }\end{array}$ & Khajing & YR & $\mathrm{C}$ & $50-100 / \mathrm{kg}$ & River, lakes \\
\hline \multicolumn{8}{|c|}{ IV. Fresh Water Mussel Variety } \\
\hline 1 & Unionidae & Anodonta anatina (Linnaeus) & Kongreng & W & $\mathrm{C}$ & $40-50 / \mathrm{kg}$ & River \\
\hline \multicolumn{8}{|c|}{ V. Fresh Water Gastropod Varieties } \\
\hline 1 & Pilidae & Pila globossa (Swainson) & Labuk tharoi & W & $\mathrm{C}$ & $10-15 / \mathrm{kg}$ & $\begin{array}{l}\text { Pond, river, paddy } \\
\text { fields }\end{array}$ \\
\hline 2 & Pilidae & Pila spp. & Tharoi ningrabi & W & $\mathrm{C}$ & $5-10 / \mathrm{kg}$ & River, wetlands \\
\hline 3 & Turritellidae & Turittella spp. & Laitharoi & W & $\mathrm{R}$ & $20-30 / \mathrm{kg}$ & River \\
\hline
\end{tabular}

S - Summer; W - Winter; YR - Year round; C - Common; R - Rare.

which in turn may lead to an increase in food intake (Sharpe 1987; Ogle 1990). Due to its high nutritive value, people throng to the markets everyday to buy these bioresources for their consumption.

Some of the wild plants and fish varieties are not only used as food items but they are also related to traditional ceremonies. Some wild plant species such as Mangifera indica Linn., Ficus glomerata Roxb., Tamarindus indica Linn., Baccaurea sapida Roxb., and Artocarpus heterophyllus Lamk are connected with traditional biofolklore and ceremonies and thus regarded as sacred species (Singh et al. 1996; Khumbongmayum et. al. 2004). Not only plants, some fish varieties also have traditional values and thus are in high demand and very 
costly. For example Eutropiichthys vacha locally called "Ngahei" is associated with the "Chaumba" ceremony, which is the first rice eating ceremony of a child. It is believed that feeding "Ngahei Curry" to the child will increase his or her knowledge and wisdom. It costs around Rs. 250-350 /kg but it can go up to Rs. 500-700 / $\mathrm{kg}$ in off-season. Similarly, Osteobrama belanger locally called "Pengba" is associated with the "Ningol Chakouba" Festival, celebrated to strengthen the bonds of love between brothers and sisters. Due to its high nutritive value and high demand it costs around Rs. 300-500 /kg. But these fish species have become so scarce that they are hardly available in the market nowadays.

Due to rapid destruction of forests and the filling up of wetlands for expansion of land area and developmental activities, most of these wild bio-resources are becoming rare and threatened. It is expected that an ever growing population will eventually lead to a greater demand for food and other necessities. As a result the existing biotic pressures may be increased several times in the near future (Sundriyal et al. 2004).

Therefore, it is important to identify the rare edible bioresources which are the livelihood of many indigenous Manipuri people so that proper conservative measures may be taken to preserve these local gene pools for our future generations.

\section{REFERENCES}

Elangbam, V.D. (2002). Studies on various aspects of wild edible plants of Manipur Valley, PhD Thesis. Department of Life Sciences, Manipur University, 148pp.

Jain, S.K. (1989). Methods and Approaches in Ethanobotany. Society of Ethnobotany, Lucknow, India, 612pp.

Jain, S.K. (1990). Contribution to Ethnobotany of India. Scientific Publishers, Jodhpur, India, pp.115-122.

Jain, S.K. (1999). Medicinal Plants. National Book Trust, India, 178pp.

Khanna, S.S. (2003). A Textbook of Fish Biology and Fisheries. Narendra Publishing House, New Delhi, 524pp.

Khumbongmayum, A.D., M.L. Khan \& R.S. Tripathi (2004). Sacred groves of Manipur - ideal centers for biodiversity conservation. Current Science 87(4): 430-432.
Martin, G.J. (1995). Ethnobotany - A Methods Manual. Chapman and Hall, London, pp.31-64.

Myers, N., R. Mittermeier, C. Mittermeier, G. da Fonseca \& J. Kent (2000). Biodiversity hotspots for conservation priorities. Nature 403: 853-858.

Mittermeier, R.A., P.R. Gil, M. Hoffmann, J. Pilgrim, T. Brooks, C.G. Mittermeier, J. Lamoreux \& G.A.B. da Fonseca (2004). Hotspots Revisited. Earth's Biologically Richest and Most Endangered Terrestrial Ecoregions. Cemex Books on Nature series, USA, 497pp.

Ogle, B.A. (1990). Dietary use of wild plant resources in rural Swaziland. Proceeding of the Twelfth Plenary Meeting of Aetfat, Symposium VIII. Mitt. Inst. Allg. Bot. Hamburg Band, 23b S. 895-910pp.

Roy, R.D. (1992). Case Study of Loktak Lake of Manipur. Wetlands of India. Ashish Publishing House, pp37-70.

Sharpe, B (1987). Report on the Nutritional Anthropology Investigations. IRDP Serenge, Mpilea, Chinsali, Isoka, Kings College, London.

Singh, H.B. \& R.K. Arora (1978). Wild Edible Plants of India. ICAR, New Delhi, India, 88pp.

Singh, P.K. (2002). Some ethnobotanically important plants available on the phumdis of Loktak Lake, pp.37-42. In: Trisal, C.L. \& Th. Manihar (eds.). Management of Phumdis in Loktak Lake.

Singh, R.N. (2006). Bioresources as economic growth of North East India, with special reference to Manipur. An article on Asia online Times, pp1-3.

Singh, B.H., P.K. Singh, S.S. Singh \& B. Elangbam (1996). Indigenous bio-folklores and practices. Its role in biodiversity conservation in Manipur. Journal of Hill Research 9(2): 359362.

Sukla, U. \& A.K. Baishya (1982). A contribution to the Flora of Manipur. Journal of Bombay Natural History Society 76: 224230.

Sundriyal, M. \& R.C. Sundriyal (2001a). Wild edible plants of Sikkim Himalaya. Nutritive values of selected species. Economic Botany 55(3): 377-390.

Sundriyal, M., R.C. Sundriyal \& E. Sharma (2004). Dietary use of wild plant resources in the Sikkim Himalayas, India. Economic Botany 58(4): 626-638.

Talwar, P.K. \& A.G. Jhingran (1991). Inland Fishes Vol 1 \& 2. Oxford and IBH Publishing Co., New Delhi, India, 1062pp.

Vishwanath, W. \& Kh. Geetakumari (2009). Diagnosis and interrelationships of fishes of the genus Channa scopoli (Teleostei. Channidae) of northeastern India. Journal of Threatened Taxa 1(2): 97- 105.

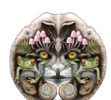

